\title{
How ordinary people in Great Britain perceive the health risks of smoking
}

\author{
Stephen Sutton
}

In a paper published in 1980 entitled "Possible ways of explaining to ordinary people the quantitative dangers of smoking" Richard Peto summarised the epidemiological evidence as follows ${ }^{1}$ : Among an average 1000 young men who smoke cigarettes regularly-about one will be murdered, about six will be killed on the roads, about 250 will be killed before their time by tobacco.

Updated figures were included in a press release that accompanied the launch in September 1994 of a book by Peto and others called Mortality from smoking in developed countries 1950-2000²: United Kingdom: current risks, on average, among 100020 year olds who smoke cigarettes regularly-about one will die from homicide, about six will die from motor vehicles, about 250 will be killed by smoking in middle age alone (+250 more in old age) ("middle" age was defined as 35-69; "old" age as 70 and over.) Corresponding figures were also given for the United States where traumatic deaths are more common than in the United Kingdom (risk of death from homicide $=6 / 1000$; risk of death from motor vehicles $=$ $12 / 1000$ ).

This way of conveying information about the quantitative risks shows strikingly just how dangerous smoking is compared with nonmedical risks. But do the general public appreciate the magnitude of the health risks from smoking? According to Peto, "In conversation, it is clear that although ordinary people know smoking to be dangerous, they have no appreciation of the quantitative risks." However, this has never been examined systematically. To my knowledge, no survey conducted in the United Kingdom has ever asked people to estimate the risks of smoking in these terms. The study reported here-part of an ESRC funded project on risk perception-attempted to remedy this by asking smokers, ex-smokers, and never-smokers to estimate the risks of being murdered, killed on the roads or killed by smoking using questions that were based closely on the statements used by Peto.

The initial sample consisted of 1968 adults aged 16 or over living in Great Britain in November 1995 recruited through the OPCS

Health Behaviour Epidemiology and Public Health, niversity College London, Brook House, 2-16 Torrington Place, Correspondence to: Dr Sutton.

Accepted for publication 30 July 1997

Table 1 Average risk estimates (out of 1000) given by smokers, ex-smokers, and never-smokers compared with epidemiological estimates

\begin{tabular}{llcccc}
\hline & & Smokers & Ex-smokers & $\begin{array}{l}\text { Never- } \\
\text { smokers }\end{array}$ & $\begin{array}{c}\text { Epidemiological } \\
\text { estimate }\end{array}$ \\
\hline Being murdered & Mean & 23 & 20 & 20 & 1 \\
Being killed on the roads & Median & 5 & 2 & 3 & \\
& Mean & 51 & 42 & 39 & 6 \\
Being killed by smoking before & Median & 15 & 10 & 10 & \\
the age of 70 & Mean & 190 & 190 & 185 & 250 \\
& Median & 100 & 100 & 100 & \\
\hline
\end{tabular}

Omnibus, a national survey that uses multistage stratified random sampling. Interviews were carried out face to face in respondents' homes using computer assisted interviewing. The response rate was $74 \%$. Exclusion of respondents who had missing or inconsistent information yielded a final sample of 1625 (764 men and 861 women). The age breakdown was: $16-249.0 \%$; 25-44 38.8\%; 45-54 $15.2 \%$; 55-64 13.9\%; 65-74 12.9\%; 75+ $10.2 \%)$. There were 465 current cigarette smokers, 425 ex-regular cigarette smokers, and 735 never-regular cigarette smokers. The prevalence of current smoking (28.6\%) was similar to that reported in the General Household Survey. ${ }^{3}$ (No information was available on the age, sex or smoking status of the nonrespondents. Compared with those retained for analysis, respondents who were excluded because of missing or inconsistent responses to the risk questions contained significantly higher proportions of women and people aged 65 and over but were similar with regard to smoking status.)

The precise wording of the risk questions was as follows: "On average, out of 100020 year olds in Britain who smoke cigarettes regularly and who carry on smoking, how many do you think will be murdered?, how many do you think will be killed on the roads?, and how many do you think will be killed by smoking before the age of 70 ?"

Respondents answered each of the three questions in turn without knowing what the next question would be. After they had answered these questions, respondents' smoking status was ascertained using the same questions that are used in the General Household Survey. ${ }^{3}$

Table 1 shows the results for the three groups of respondents (smokers, ex-smokers, neversmokers) for each of the three hazards in terms of the mean and median. The epidemiological estimates are shown for comparison. As the distributions were highly skewed, particularly for homicide, non-parametric statistical tests were used (Kruskal-Wallis one way analysis of variance and Mann-Whitney U test).

Smoking was seen as by far the most dangerous of the three hazards, followed by death on the roads, then homicide; the rank order corresponds to the epidemiological estimates. For the first two hazards (being murdered and being killed on the roads), the risk estimates given by the three groups of respondents were broadly similar, although smokers gave significantly $(\mathrm{p}<0.01)$ higher estimates than ex- and never-smokers. However, for the risk of being killed by smoking, the estimates were remarkably similar with no significant differences 

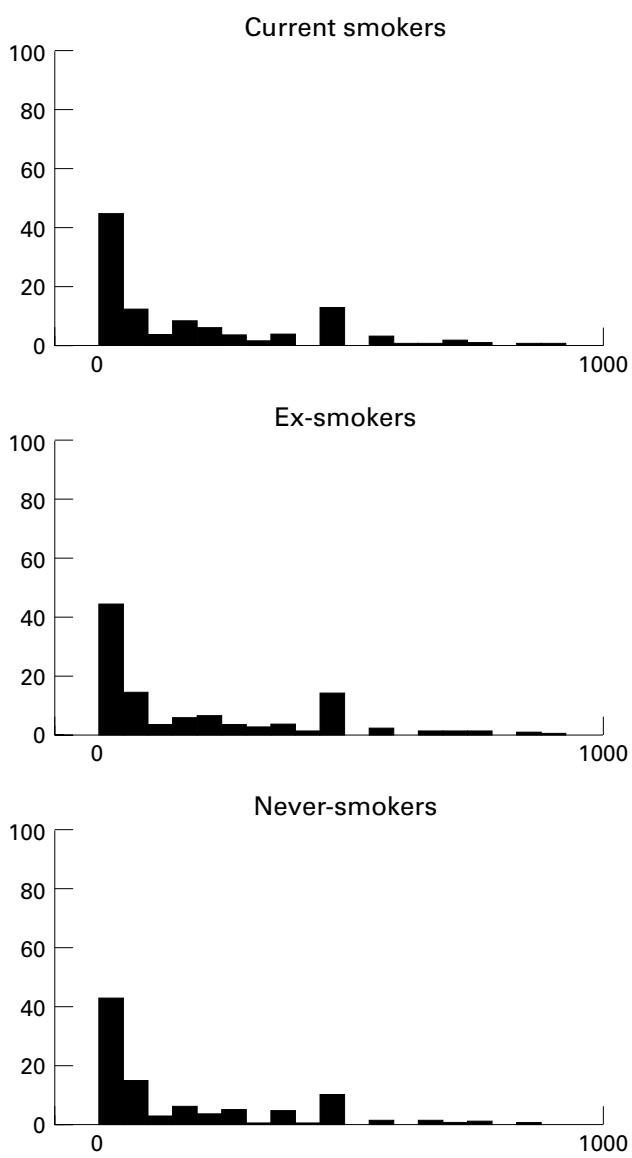

Figure 1 Relative frequency distributions for estimates of the risk of being killed by smoking before the age of 70, for three groups of respondents (current, ex-smokers, and never-smokers).

across the three groups of respondents. The distributions in figure 1 show graphically the striking similarity in response in the three groups.

Estimates of the risk of being killed by smoking were further analysed by creating 12 agesex subgroups. There were no significant differences between smokers, ex-smokers, and never-smokers in any of the subgroups. Thus controlling for age and sex does not qualify the overall conclusion that the three groups of respondents gave very similar risk estimates. There was no significant sex difference; men and women gave similar estimates of the risk of being killed by smoking. However, there was a substantial and statistically significant ( $p$ $<0.001$ ) effect of age. As figure 2 shows, the 16-24 age group had a median risk estimate of 200 compared with 100 or less in the other five age groups; the means showed a similar overall pattern. It is not clear why the youngest age group gave the highest risk estimates but it is possibly related in part to the fact that the risk question referred to " 20 year olds".

These findings suggest that, on average, smokers in Britain do appreciate that smoking is much more dangerous than other hazards and that they do so to the same extent as ex-smokers and never-smokers. There is no evidence here that smokers deny the health risks of smoking or are less knowledgeable

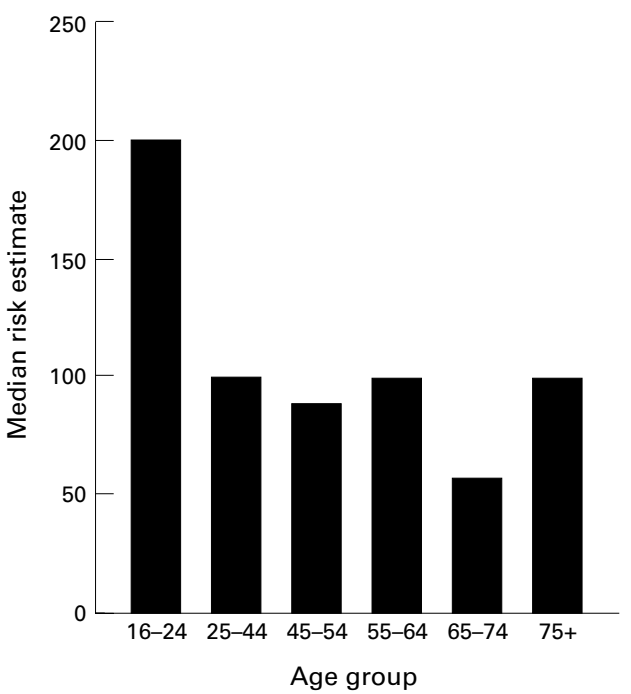

Figure 2 Median estimates of the risk of being killed by smoking before the age of 70, by age group.

about the risks than non-smokers-or that non-smokers tend to exaggerate the risks relative to smokers. On the other hand, compared with non-smokers, smokers seem to regard life (for smokers) as somewhat more dangerous in terms of the risk of traumatic death.

Although these results are encouraging, there is still much scope for improvement. Only a small minority of respondents gave estimates for the risk of being killed by smoking that came anywhere near the epidemiological point estimate of 250 in 1000. For example, if - allowing a reasonable margin of erroranswers in the range 200-300 are considered to be "correct", the vast majority $(84.5 \%)$ of respondents gave inaccurate estimates: $61.1 \%$ underestimated the risk and $23.4 \%$ overestimated it. Consistent with the findings reported above, there were no significant differences in accuracy defined in this way between current smokers, ex-smokers, and never-smokers or between men and women. Again, however, there was a significant age effect $(p<0.001)$. The over 65 age group had the lowest accuracy rate $(12.5 \%)$ and the $16-24$ and $55-64$ age groups had the highest rates $(18.4 \%$ and $19.9 \%$ respectively).

It is important that smoking education campaigns and materials continue to present clear and accurate quantitative information about the health risks of smoking. As Peto suggests, ${ }^{1}$ a brief statement comparing the risks of smoking with those of other familiar hazards could be used as a warning on cigarette packets and advertisements. Funding: this research was funded by the Economic and Social
Research Council (ESRC) under the Risk and Human Behaviour Research Programme (Grant No. L211252028) and by the Imperial Cancer Research Fund (ICRF).

1 Peto R. Possible ways of explaining to ordinary people the quantitative dangers of smoking. Health Educ $\mathcal{f}$ 1980;39:45-6.

2 Peto R, Lopez AD, Boreham J, et al. Mortality from smoking in developed countries 1950-2000. Oxford: Oxford University Press, 1994

3 Thomas M, Goddard E, Hickman M, et al. General household survey 1992. London: HMSO, 1994. 